\title{
Repeated Moderate Noise Exposure in the Rat-an Early Adulthood Noise Exposure Model
}

\author{
Paula Mannström, ${ }^{1}$ (D Mette KirkegaArd, ${ }^{1}$ and Mats Ulfendahl ${ }^{1}$ \\ ${ }^{1}$ Department of Neuroscience, Karolinska Institutet, Retzius väg 8, B1:5, SE-171 77, Stockholm, Sweden
}

Received: 17 June 2014; Accepted: 26 June 2015; Online publication: 11 July 2015

\begin{abstract}
In this study, we investigated the effects of varying intensity levels of repeated moderate noise exposures on hearing. The aim was to define an appropriate intensity level that could be repeated several times without giving rise to a permanent hearing loss, and thus establish a model for early adulthood moderate noise exposure in rats. Female Sprague-Dawley rats were exposed to broadband noise for $90 \mathrm{~min}$, with a $50 \%$ duty cycle at levels of $101,104,107$, or $110 \mathrm{~dB}$ sound pressure level (SPL), and compared to a control group of non-exposed animals. Exposure was repeated every 6 weeks for a maximum of six repetitions or until a permanent hearing loss was observed. Hearing was assessed by the auditory brainstem response (ABR). Rats exposed to the higher intensities of 107 and $110 \mathrm{~dB}$ SPL showed permanent threshold shifts following the first exposure, while rats exposed to 101 and $104 \mathrm{~dB}$ SPL could be exposed at least six times without a sustained change in hearing thresholds. ABR amplitudes decreased over time for all groups, including the non-exposed control group, while the latencies were unaffected. A possible change in noise susceptibility following the repeated moderate noise exposures was tested by subjecting the animals to high-intensity noise exposure of $110 \mathrm{~dB}$ for $4 \mathrm{~h}$. Rats previously exposed repeatedly to $104 \mathrm{~dB}$ SPL were slightly more resistant to high-intensity noise exposure than non-exposed rats or rats exposed to $101 \mathrm{~dB}$ SPL. Repeated moderate exposure to $104 \mathrm{~dB}$ SPL broadband noise is a viable model for early adulthood noise exposure in rats and may be useful for the study of noise exposure on age-related hearing loss.
\end{abstract}

Correspondence to: Paula Mannström · Department of Neuroscience · Karolinska Institutet · Retzius väg 8, B1:5, SE-171 77, Stockholm, Sweden. Telephone: +46 8524869 44; email: paula.mannstrom@ ki.se
Keywords: repeated noise exposure, hearing, threshold shift, amplitude, rat

\section{INTRODUCTION}

Hearing impairment is one of the most common disabilities in modern society, according to National Institute on Deafness and Other Communication Disorders (NIDCD, 2010). Of the US adult population, $17 \%$ report some degree of hearing loss, a number which increases with age. Genetic factors contribute significantly to the functional impairment, but external environmental factors such as exposure to intense noise (Oishi and Schacht 2011), ototoxic drugs (Rybak et al. 2007), or solvent chemicals (Johnson and Nylen 1995) are equally important and may exacerbate the hearing loss. Increased awareness of risk factors has resulted in protective measures, and today, the working population is to a much lesser extent subjected to intense noise at the workplace. However, we are living in an increasingly noisy world where individuals are exposed to moderate everyday noise from their leisure activities, e.g., loud music from earphones and high volume at the cinemas, gyms, rock concerts, etc. While these repeated noise exposures, sometimes of quite high intensities, usually do not elicit a detectable loss of hearing, their longterm effects on auditory function are not well explored. It has, however, been suggested that these exposures will adversely affect the progression of agerelated hearing loss (Stockwel et al. 1969; Johnsson and Hawkins 1972; Mostafapour et al. 1998; Dalton et al. 2001).

The effect of noise exposure on hearing has been studied extensively in mammals (Liberman and 
Dodds 1984; Slepecky 1986; Wang et al. 2002). Typically, intense noise results in a permanent hearing loss, while noise at lower intensities causes a temporary hearing loss. In both cases, the hearing loss reflects oxidative stress and structural changes at the level of the hearing organ. In conditions of permanent threshold shift, mechanical damage to the inner and outer hair cells as well as the afferent nerve fibers has been reported (for a review, see Ohlemiller 2008). The cellular damage also leads to a secondary degeneration of neurons (Kujawa and Liberman 2006), and moreover, stria vascularis and fibrocytes in the spiral ligament are affected (Hirose and Liberman 2003). In cases of a temporary threshold shift, structural observations include degeneration of afferent nerve terminals, detachment of the hair cell stereocilia from the overlying tectorial membrane, and buckling of pillar cells (Nordmann et al. 2000). Those changes are explained as direct consequences of moderate noise exposures. Other consequences are swelling of cochlear nerve terminals, which is an acute response to noise caused by glutamate excitotoxicity. Loss of presynaptic ribbons and postsynaptic terminals of the inner hair cells precedes the swelling (Kujawa and Liberman 2009; Lin, et al. 2011; Ruttiger et al. 2013). These changes remain even when the hearing thresholds return to normal levels. Interestingly, in these studies the auditory brainstem response (ABR) amplitudes did not recover, which were believed to be a consequence from the histopathological changes of the peripheral nerve. Also, the biochemical composition becomes altered after noise exposures. For example, both excitatory and inhibitory neurotransmission-related proteins and neuroplasticity markers were altered in the rat auditory pathway after intense noise exposure (Browne et al. 2012).

The degree of hearing loss after noise exposure depends on the total energy content of the noise, which in turn depends on the noise intensity, exposure time, possible repetition rate, and interval length as described in a review article by Clark (Clark 1991). By integrating periodic rest periods, some protective effects were observed in terms of less hearing loss and histological damage. In a study by Clark and colleagues (Clark et al. 1987), chinchillas were exposed to octave band noise, $95 \mathrm{~dB}$ sound pressure level (SPL), $6 \mathrm{~h}$ per day for 36 days. The initial threshold shift of 35-45 dB recovered after a few days of exposure to a shift of 10-15 dB. In another study (Hamernik et al. 1994), more intense noise exposures (107, 113, 119, and $125 \mathrm{~dB}$ SPL) were used at two different exposure protocols in chinchillas. One group was exposed $6 \mathrm{~h}$ per day for 20 days while another group was exposed during a non-interrupted schedule of $24 \mathrm{~h}$ per day for a total of 5 days. Both groups thus received the same total exposure energy. Hearing recovered better and histological data revealed a milder hair cell loss for the group exposed on an interrupted schedule over a longer period. This data suggests a toughening of the hearing organ when rest periods are introduced to the noise exposure protocol.

Kujawa and Liberman showed that noise exposure in young $\mathrm{CBA} / \mathrm{CaJ}$ mice exacerbated the age-related hearing loss in aging animals, compared to nonexposed age-matched controls, even if the initial increased threshold shift after the noise exposure was reversible (Kujawa and Liberman 2006). Interestingly, when a group of older animals was exposed to the same type of noise, the initial effects on hearing thresholds as well as the structural degeneration of the neurons and the sensory cells were not as severe, as in the younger animals. This study indicates that a reversible hearing loss at a young age may accelerate the age-related hearing loss and that younger animals are more sensitive to noise than older ones. It is therefore of interest and clinical importance to study also the effect of moderate noise exposure in younger animals and the long-term effects on hearing.

The aim of the present study was to develop an animal model mimicking the exposure of young adults to non-damaging noise levels, by exposing rats in early adulthood to repeated moderate noise at levels that do not cause a permanent hearing loss. The effects of different intensity levels were evaluated by monitoring changes in ABR. Female SpragueDawley rats were exposed to noise at selected intensities at 6-week intervals, up to six times or until they displayed a permanent threshold shift. The groups that did not show permanent threshold shifts even after six exposures were subjected to a high-intensity noise exposure to assess the influence of repeated noise preexposures on the subsequent susceptibility to noise-induced pathology. The main objectives of the present study was to explore how an increase in intensity levels of repeated noise exposures changed the threshold shift in an animal from temporary to permanent. This included monitoring the functional changes of ABR threshold shifts, reduced amplitudes, and delayed latencies. Finally, it was of interest to investigate whether repeated exposures to moderate noise altered the susceptibility to more intense noise.

\section{MATERIAL AND METHODS}

\section{Subjects}

A total of 40 female albino Sprague-Dawley rats (Harlan laboratories, the Netherlands) starting from the age of 9 weeks were used in this study. Animals were kept three to four in wire-meshed cages with free 
access to food and water in the same holding unit on a 12/12-h day and night cycle. All animal procedures followed the local ethical regulations at Karolinska Institutet and were in consistence with national regulations for care and use of animals, approval numbers N33/07, N12/10, and N300/11.

\section{Experimental Design}

The animals were divided into groups exposed to brief periods of noise at different intensities: 101 $(n=8), 104(n=8), 107(n=7)$, and $110(n=7)$ dB SPL. Age-matched animals that were not exposed served as a control group $(n=10)$. Exposures were repeated every 6 weeks for up to six times or until the animals received a permanent hearing loss (Fig. 1). Baseline hearing thresholds were assessed by recording ABR 1 week before the first noise exposure. The ABRs were then recorded $24 \mathrm{~h}, 1$ week, 2 weeks, and 5 weeks after each repeated exposure. For the non-exposed control animals, ABRs were recorded once every 6 weeks from 9 weeks of age. The groups which displayed no permanent threshold shift even after six repetitions and the non-exposed group were finally exposed to a high-intensity noise, known to cause permanent threshold shifts in this animal strain (unpublished data), and the hearing was assessed $24 \mathrm{~h}$ and 1 and 2 weeks following the intense exposure.

\section{Noise Exposures}

Two freely moving rats were simultaneously exposed in individual cages, centrally placed side by side inside a soundproof box with the dimensions $104 \times 78 \times 112 \mathrm{~cm}$ (width $\times$ depth $\times$ height). The noise, generated using Brüel \& Kjær 3560-C PULSE hardware and a LAB 300 amplifier with PULSE LabShop Version 13.1.0.246 software (Brüel \& Kjær, Denmark), was presented through a transducer (Model 2482, JBL, LA, USA) with a Beyma TD-360 horn (Acustica Beyma, Spain) centrally positioned inside the box, $85 \mathrm{~cm}$ above the animal cages. The noise was calibrated using a microphone (Brüel \& Kjær, Denmark) placed between the cages and adjusted to the desired level before each exposure. Prior to the experiments, the noise level was recorded at each of the four corners of the cages and shown to vary by $\pm 2 \mathrm{~dB}$ SPL.

For repeated moderate noise exposures, the rats were exposed to broadband free-field noise, 2$20 \mathrm{kHz}$, at a $50 \%$ duty cycle $(500 \mathrm{~ms}$ of exposure each second) for $90 \mathrm{~min}$ at intensity levels of 101, 104, 107 , or $110 \mathrm{~dB}$ SPL. Groups that showed no permanent threshold shift after six repetitions (i.e., the 101 and the $104 \mathrm{~dB}$ SPL groups) and the non-exposed control group were then exposed to a high-intensity noise of $110 \mathrm{~dB}$ SPL (narrowband free-field noise, $3.2 \mathrm{kHz}$ bandwidth) for $4 \mathrm{~h}$.

\section{Hearing Assessment by Recording Auditory Brainstem Responses}

The animals were anesthetized using an intraperitoneal injection of a $2.7 \mathrm{ml} / \mathrm{kg}$ mixture of $1 \mathrm{ml}$ Hypnorm ${ }^{\circledR}, 1 \mathrm{ml}$ Dormicum ( $5 \mathrm{mg} / \mathrm{ml}$ Midazolam ${ }^{\circledR}$ ), and $2 \mathrm{ml}$ sterile water. ABR recordings were randomly made in either left or right ear, and the same ear was subsequently used throughout the study. Before the first ABR recording was performed, the tragus cartilage was removed in order to place the microphone close to the eardrum. The eardrums were inspected using an otoscope, and animals with middle ear infections were excluded. After placing the animal on a non-electric heating pad inside a soundproof box, subcutaneous needle electrodes were positioned at the vertex (active), behind the recorded ear (reference), and in the hind leg (ground). Acoustic stimuli were generated by a Tucker-Davis Technologies system (BioSig 32 Ver 3.12, Tucker-Davis Technologies, FL, USA), presented through an electrostatic speaker (EC1, Tucker-Davies Technologies, FL, USA), which was connected to the ear canal of the rat. Frequency-specific tone bursts of 3.5, 7, 14, and $28 \mathrm{kHz}$ were presented at a duration of $2,1,0.5$, and $0.25 \mathrm{~ms}$ for the respective frequencies and with a $1,0.5,0.25$, and $0.125 \mathrm{~ms}$ raise and fall time.

ABR thresholds were determined as the lowest stimulus level that produced a reproducible response for $A B R$ wave II, which was visualized at the same latency after an average of 2000 recordings. The second wave was chosen as it is the largest wave of the rat's $A B R$ and thus most readily monitored, also when the stimulus level is lowered. The stimulus intensity was lowered in steps of minimum $5 \mathrm{~dB}$ until the threshold was reached. The threshold shifts were calculated by subtracting the recorded thresholds with the baseline threshold recorded before the first exposure. Amplitudes and latencies from ABR wave I were extracted at an acoustic stimulus of $80 \mathrm{~dB}$ SPL after an average of 1000 recordings. Wave $\mathrm{I}$ is generated from the auditory nerve and is a measure of the number and the synchrony of the neurons firing. The amplitudes were measured as the peak to peak ${ }_{(p-p)}$ amplitude difference from the peak of wave I (P1) to its trough (T1) (Fig. 2) (Alvarado et al. 2012). The latencies were defined as the time between the onset of the stimulus and the peak of wave I (P1) (Fig. 2). The relative amplitudes and latencies were calculated by dividing the value at each time point with the value from the first measurement. Measurements were made at all measured frequencies. 


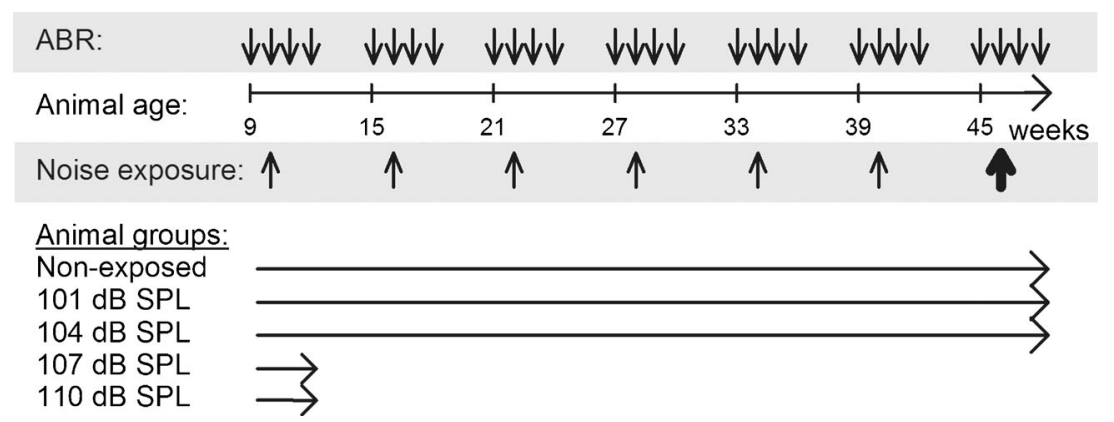

FIG. 1. The experimental design showing time points for $A B R$ recordings (downward arrows) 1 week before the first noise exposure and $24 \mathrm{~h}, 1$ week, 2 weeks, and 5 weeks after each of the repeated moderate noise exposures (thin upward arrows) followed by high-

\section{Statistics}

Differences between groups were compared statistically by one-way ANOVA followed by a Holm-Sidak comparison, and comparisons within the groups over time were analyzed using one-way repeated measures ANOVA followed by Holm-Sidak comparison on repeated measurements. When normality test failed, Kruskal-Wallis one-way ANOVA on ranks using Dunn's method as an all pair-wise multiple comparison procedure was used. The significance level was set to $P<0.05$. Data analysis was performed using SigmaPlot for Windows Version 11.0. All data are presented as mean \pm standard deviation $(\mathrm{SD})$.

\section{RESULTS}

A Single Exposure at 107 or $110 \mathrm{~dB}$ SPL Elicited a Permanent Threshold Shift

After the first exposure to moderate noise, the rats' auditory function was affected. For animals exposed to 107 or $110 \mathrm{~dB}$ SPL, ABR threshold shifts were around $20 \mathrm{~dB}$ (for all tested frequencies) at $24 \mathrm{~h}$ after the first exposure (Fig. 3A). At $7 \mathrm{kHz}$, these groups differed significantly from animals exposed to 101 and $104 \mathrm{~dB}$ SPL (one-way ANOVA on ranks, $H_{(3)}=18.6$, $P<0.05$ for both $110 \mathrm{~dB}$ compared to 101 and $104 \mathrm{~dB}$ SPL as well as $107 \mathrm{~dB}$ SPL compared to $101 \mathrm{~dB}$ SPL). After 2 weeks, all threshold shifts decreased in all subjects, but the animals exposed to 107 and $110 \mathrm{~dB}$ SPL continued to display an increased threshold shift of about $15 \mathrm{~dB}$ at all tested frequencies (Fig. 3B), and the shifts were thus considered permanent. The threshold shifts were significantly higher (one-way ANOVA at $\left.3.5 \mathrm{kHz} ; F_{(3)}=16.2, P<0.001\right)$ both for 107 and $110 \mathrm{~dB}$ SPL compared to 101 and $104 \mathrm{~dB}$ SPL. At $7 \mathrm{kHz} ; F_{(3)}=12.6, P<0.001$ for $107 \mathrm{~dB}$ SPL compared to 101 and $104 \mathrm{~dB}$ SPL, $P=0.001$ for $110 \mathrm{~dB}$ SPL compared to $101 \mathrm{~dB}$ SPL and $P=0.004$ for $110 \mathrm{~dB}$ SPL compared to $104 \mathrm{~dB}$ SPL. At $14 \mathrm{kHz}$ one-way ANOVA intensity noise exposure (thick upward arrow). The horizontal arrows after the five animal groups correspond to time spent in the experiment.

on ranks, $H_{(3)}=9.77, P<0.05$ for $107 \mathrm{~dB}$ SPL compared to $104 \mathrm{~dB}$ SPL. At $28 \mathrm{kHz}$ one-way ANOVA on ranks, $H_{(3)}=8.71, P<0.05$ for $107 \mathrm{~dB}$ SPL compared to $104 \mathrm{~dB}$ SPL) than those obtained in animals exposed to the lower intensities (101 and $104 \mathrm{~dB}$ SPL), which had a mean threshold shift of about $2 \mathrm{~dB}$. The animals exposed to 104 or $101 \mathrm{~dB}$ SPL differed neither from each other nor from the non-exposed control group regarding threshold shifts.

104 dB SPL Was the Highest Intensity Level that Could Be Repeated Six Times Without Causing a Permanent Threshold Shift

The animals exposed to intensity levels of 107 and 110 dB SPL displayed permanent threshold shifts after the first exposure, and these groups were thus not further exposed. The remaining groups, exposed to intensity levels of 101 and $104 \mathrm{~dB}$ SPL, could be exposed for at least six repetitions without achieving permanent threshold shifts (average threshold shifts

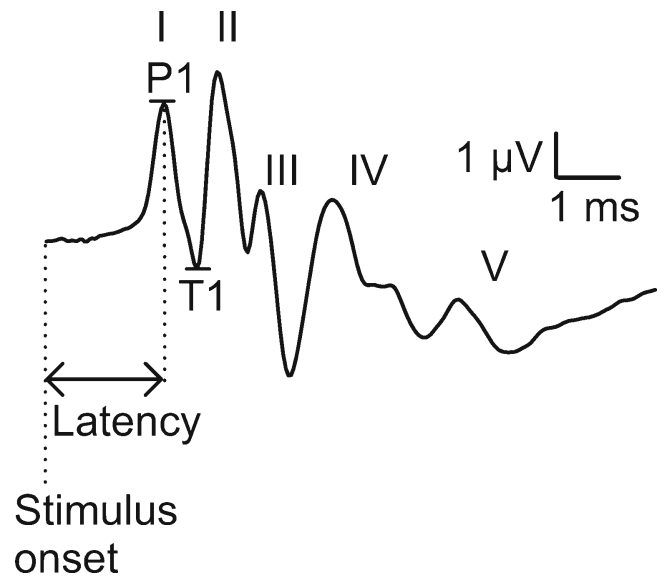

FIG. 2. ABR waveforms marked $l-V$ from a young rat with normal threshold. ABR wave $\mathrm{I}_{p-p}$ amplitudes $(\mu \mathrm{V})$ were calculated as the peak to peak difference from wave I peak (P1) to its trough (T1). The dotted lines indicate the latency, i.e., the time from the onset of the stimuli to the peak of wave I. 


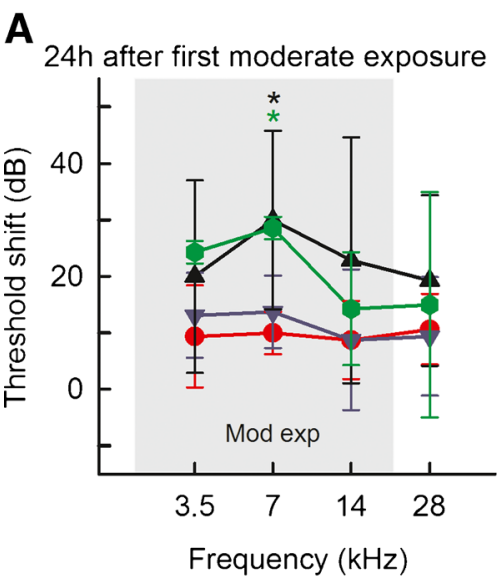

FIG. 3. A ABR threshold shifts $24 \mathrm{~h}$ after the first moderate noise exposure. Significantly increased thresholds of 15-28 dB for the groups exposed to 107 and $110 \mathrm{~dB}$ SPL compared to the groups exposed to 101 and $104 \mathrm{~dB}$ SPL. B Two weeks later, the threshold shifts for the 107 and $110 \mathrm{~dB}$ SPL groups were reduced to $11-20 \mathrm{~dB}$ but were still significantly higher than for the groups exposed to 101

from a representative $\mathrm{ABR}$ frequency from $7 \mathrm{kHz}$ is shown in Fig. 4). Each exposure produced a temporary threshold shift of approximately $10 \mathrm{~dB}$ at $24 \mathrm{~h}$ and 1 week after the noise exposure, but after 2 weeks, the ABR thresholds returned to baseline levels. All tested frequencies displayed the same pattern. The non-exposed control group displayed stable thresholds throughout the whole period, thus confirming that no age-related hearing loss affected the hearing during the experiment. The animals were 45 weeks old at the time point for the last ABR recording.

\section{ABR Amplitudes Were Reduced in Animals with Recovered Thresholds}

For the groups exposed to the lower intensity levels (101 and $104 \mathrm{~dB}$ SPL), the effect of the repeated moderate noise exposures were explored in detail

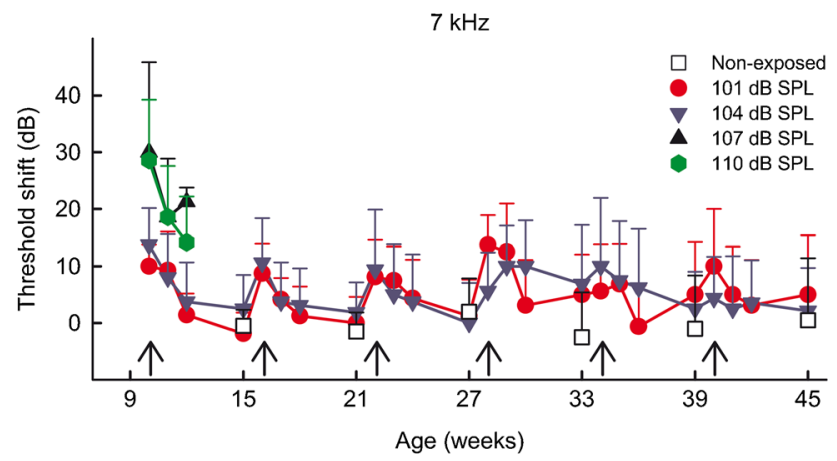

FIG. 4. Mean ABR threshold shifts at $7 \mathrm{kHz}$ for the animal groups exposed to different intensity levels $(101,104,107$, and $110 \mathrm{~dB}$ SPL) at $24 \mathrm{~h}, 1$ week, 2 weeks, and 5 weeks after each repeated noise exposure. In the age-matched, non-exposed control group, ABRs were recorded every 6 weeks. Arrows indicate the time points for the repeated exposures.

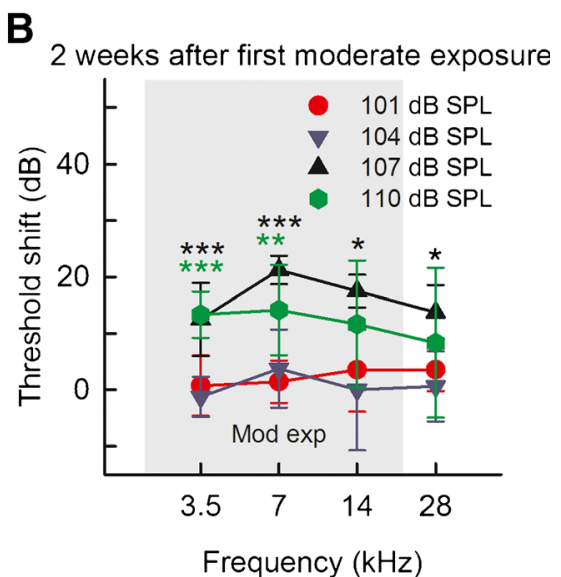

and $104 \mathrm{~dB}$ SPL, where the thresholds had returned to baseline levels. The area marked Mod exp is the frequency span for the moderate exposure. ${ }^{*} P<0.05,{ }^{* *} P<0.01$, ${ }^{* * *} P<0.001$, colors correspond to exposure groups, which significantly differ from the other groups.

5 weeks after each exposure (i.e., every 6 weeks) and compared to the non-exposed control group (Fig. 5). Threshold shifts as well as the ABR wave I $p-p$ amplitudes and latencies were compared between the groups to investigate the impact of the different intensity levels. Effects of the repeated exposures and age changes were also studied by comparing ABRs within the same group over time. The ABR threshold shifts in all three groups varied only with $\pm 5-10 \mathrm{~dB}$ 5 weeks after each exposure, both between the groups and over time within the groups, which was within the limit of individual differences (the $101 \mathrm{~dB}$ SPL group at representative measurements from $7 \mathrm{kHz}$ is shown in Fig. 5A). Neither the repeated moderate exposures nor age had any effect on the threshold shifts. When comparing ABR wave I $p$ p amplitudes, no significant differences were detected between the groups at any time point. However, significant reductions were detected within all three groups over time. The $101 \mathrm{~dB}$ SPL group displayed significantly reduced amplitudes after the first exposure and at all time points thereafter (one-way repeated measures ANOVA, $F_{(6)}=7.34$, Holm-Sidak test for comparison over time, representative measurements from $7 \mathrm{kHz}$ shown in Fig. 5B and Table 1). The ABR amplitudes of the group exposed to $104 \mathrm{~dB}$ SPL were also reduced, but significant changes were not detected until the measurement after the fourth exposure (one-way repeated measures ANOVA, $F_{(6)}=2.78$, Holm-Sidak test for comparison over time, representative measurements from $7 \mathrm{kHz}$ shown in Fig. 5B and Table 1). Interestingly, in the non-exposed control group significantly reduced amplitudes were detected at the time point when the exposure groups were measured after their third exposure (one-way repeat- 


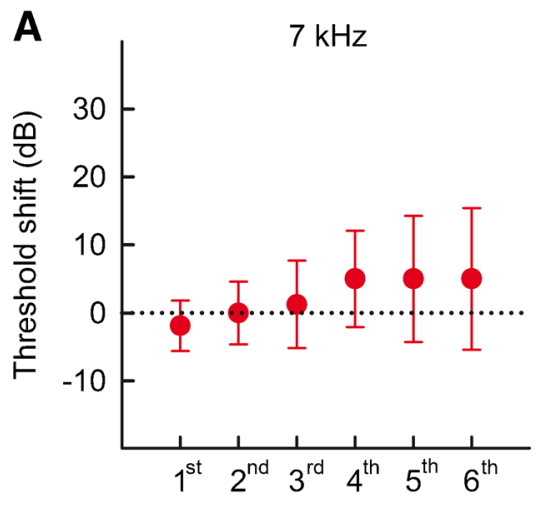

After moderate exposure

FIG. 5. A No significant differences in $A B R$ threshold shifts were seen over time when comparing ABRs 5 weeks after each of the repeated moderate noise exposures. ABRs recorded at $7 \mathrm{kHz}$ for the group exposed to $101 \mathrm{~dB}$ SPL is shown. B Relative ABR wave I $p$-p amplitudes were significantly reduced over time compared to the

ed measures ANOVA, $F_{(6)}=4.909$, Holm-Sidak test for comparison over time, representative measurements from $7 \mathrm{kHz}$ shown in Fig. 5B and Table 1). Amplitudes were thus reduced with age rather than as an effect of the repeated moderate noise exposures.

The ABR latencies were more stable over time, and only in few of the measurements, after several exposures, prolonged latencies were detected in the group exposed to $104 \mathrm{~dB}$ (data not shown). Also here, no statistical differences were found between the groups.

Repeated Exposures at $104 \mathrm{~dB}$ SPL Resulted in Animals Appearing More Resistant to HighIntensity Noise Exposure

Even though no permanent hearing thresholds were observed in the groups repeatedly exposed to 101 and $104 \mathrm{~dB}$ SPL, a possible change in noise susceptibility was investigated. The animals, including the previously non-

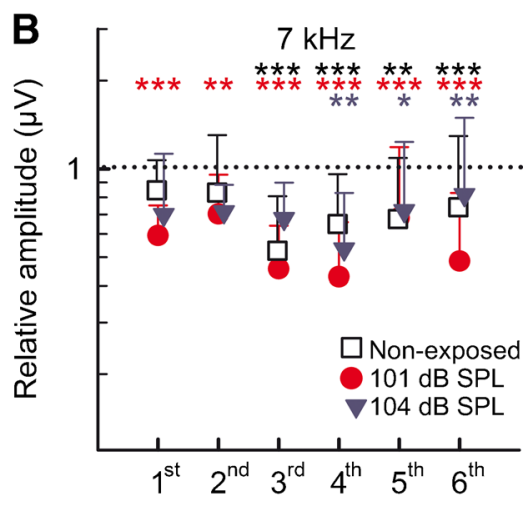

After moderate exposure

amplitudes from the first ABRs before the first exposure. Dotted lines indicate baseline values. ${ }^{* *} P<0.01,{ }^{* * *} P<0.001$, compared to baseline values within the same group, colors correspond to exposure groups.

exposed group, were thus exposed to $110 \mathrm{~dB}$ SPL narrowband noise for $4 \mathrm{~h}$, an exposure known to induce permanent threshold shifts in this rat strain. The resulting threshold shifts peaked after the more intense exposure to around $40-50 \mathrm{~dB}$ at $24 \mathrm{~h}$ following the exposure (Fig. 6A), decreased with time, and was permanent at about 20-40 dB after 2 weeks (Fig. 6B). The highest threshold shift was found at the lower frequencies, close to frequency of the noise exposure. The group previously exposed repeatedly to $104 \mathrm{~dB}$ SPL displayed slightly lower threshold shifts compared to the other groups at all recorded time points after the acoustic overstimulation, statistically significant after 2 weeks at $28 \mathrm{kHz}$ when the thresholds where back to normal levels (one-way ANOVA, $F_{(2)}=3.95$, compared to the previously non-exposed group; Holm-Sidak test, $P=0.013)$. Neither the wave I $p p$ amplitudes nor latencies of the ABR wave differed at any time point between any of the groups (data not shown).

TABLE 1

Relative ABR wave I ${ }_{p-p}$ amplitudes after each repeated moderate noise exposure (values divided with the value from the first measurement)

\begin{tabular}{llll}
\hline After moderate exposure & $\begin{array}{l}\text { Non-exposed } \\
n=10, F_{(6)}=4.91\end{array}$ & $\begin{array}{l}101 \mathrm{~dB} S P L \\
n=8, F_{(6)}=7.34\end{array}$ & $\begin{array}{l}104 \mathrm{~dB} S P L \\
n=8, F_{(6)}=2.78\end{array}$ \\
\hline 1 & $0.83 \pm 0.22$, n.s. & $0.59 \pm 0.16, P<0.001$ & $0.70 \pm 0.43$, n.s. \\
2 & $0.82 \pm 0.47$, n.s. & $0.70 \pm 0.26, P=0.01$ & $0.72 \pm 0.17$, n.s. \\
3 & $0.52 \pm 0.28, P<0.001$ & $0.46 \pm 0.18, P<0.001$ & $0.67 \pm 0.22$, n.s. \\
4 & $0.64 \pm 0.31, P=0.001$ & $0.43 \pm 0.23, P<0.001$ & $0.53 \pm 0.30, P=0.002$ \\
5 & $0.67 \pm 0.41, P=0.007$ & $0.68 \pm 0.51, P<0.001$ & $0.72 \pm 0.52, P=0.012$ \\
6 & $0.73 \pm 0.51, P=0.001$ & $0.48 \pm 0.34, P<0.001$ & $0.82 \pm 0.69, P=0.005$ \\
\hline
\end{tabular}

Average levels \pm standard deviations with significance level (one-way repeated measures ANOVA, Holm-Sidak test compared to the baseline value from the same exposure group) for the different groups, ABRs at $7 \mathrm{kHz}$ with a stimulus level of $80 \mathrm{~dB} \mathrm{SPL}$

n.s. not significant 


\section{DISCUSSION}

In this study, we investigated the functional effects of moderate noise exposures at different intensity levels in early adulthood of the female Sprague-Dawley rat in order to determine the levels that could be repeated several times without causing permanent threshold shifts. In the present model, $104 \mathrm{~dB}$ SPL was the highest exposure intensity that could be repeated up to six times without causing a permanent change in hearing thresholds. In addition, exposures at this level provided a certain protective effect from subsequent acoustic overstimulation of higher-intensity noise exposure. We suggest that repeated exposures of $104 \mathrm{~dB}$ SPL can be used as a model for studying the influence of early adulthood noise exposures on agerelated hearing loss.

A broadband noise exposure with intensity levels of both 101 and $104 \mathrm{~dB}$ SPL, for $90 \mathrm{~min}$ at a $50 \%$ duty cycle, could be repeated up to six times with no permanent effect on hearing thresholds. The same type of exposures with intensity levels of 107 or $110 \mathrm{~dB}$ SPL produced a permanent threshold shift following the first exposure. The exposures started when the female rats were young adults (10 weeks old) and continued until the rats were middle-aged (about 10 months of age). At this age, the non-exposed rats displayed normal hearing thresholds without any signs of an incipient age-related hearing loss. It is possible that the first exposure could fall within an early vulnerable period for the rat. However, previous studies indicate that the most sensitive period for the rat are around 20-25 days (Lenoir et al. 1979, Saunders 1982), which corresponds to the period of functional and structural maturation of the cochlea. In the present study, the first exposure started at 10 weeks of age and, at that time, the cochlea was fully mature and the early vulnerable period had passed. The onset of age-related hearing loss in this strain (Sprague-Dawley) occurs around 12 months of age. The Sprague-Dawley rat is an outbred strain and was chosen for this study because of its similarities to humans regarding diversity in sensory and motor function during aging (Altun et al. 2007) and with the aim to be used as a future model strain. The age-related histological changes of the inner ear in the SpragueDawley rat have previously been studied in our laboratory. Loss of sensory hair cells and a diminished volume of stria vascularis were the major age-related changes with increasing age (Mannstrom et al. 2013). In the future, both histological as well as biochemical changes need to be further explored after repeated moderate noise exposures and increasing age.

Threshold shifts, wave I $p$ p amplitudes, and latencies were determined from ABRs that were conducted 5 weeks after each of the repeated moderate noise exposures in the animal groups that did not demonstrate a permanent threshold shift after the exposures, e.g., the groups exposed to 101 and $104 \mathrm{~dB}$ SPL, together with the non-exposed group. The thresholds were normal both over time and between all groups. The ABR wave I ${ }_{p-p}$ amplitudes decreased significantly over time for all groups, including the control group, but did not significantly differ between the groups. This indicates that the spiral ganglion activity, reflected in the magnitude of the ABR amplitude, was reduced due to age rather than exposure. The decline was significant compared to baseline levels after the first exposure for the group exposed to $101 \mathrm{~dB}$ SPL and after the fourth exposure for the group exposed to $104 \mathrm{~dB}$ SPL. The animals in the control group were almost 7 months (27 weeks) when their amplitudes were significantly reduced; if compared to humans, it would correspond to early midlife. In human studies, the peak to peak amplitude of the ABR waves also becomes reduced at early ages, when hearing thresholds are still normal (KonradMartin et al. 2012). Consistent with other animal studies (gerbils and rhesus monkeys), we found that amplitudes were reduced with age irrespectively of threshold shifts (Boettcher et al. 1993; Torre and Fowler 2000). In addition to increasing age, the most common reason for a decline in amplitude is noise exposures. In a study where guinea pigs were exposed to octave band noise $(4-8 \mathrm{kHz}$, for $2 \mathrm{~h}$ at $106 \mathrm{~dB}$ SPL) eliciting a temporary threshold shift, permanent reduced amplitudes as well as structural changes were observed (Lin et al. 2011). In another study, using repeated noise exposures, the same pattern of altered amplitudes preceding permanent threshold shift changes was presented (Wang and Ren 2012). In that study, CBA/CaJ mice were exposed three times to a $100 \mathrm{~dB}$ SPL noise $(8-16 \mathrm{kHz}, 2 \mathrm{~h}, 2$ weeks apart). The ABR wave I $p-p$ amplitudes were reduced after the first noise exposure, but only after the third exposure the threshold shift became permanent. In contrary to these studies, we did not detect any significantly altered amplitudes caused by the exposures creating temporary threshold shift. The reduced amplitudes were instead considered as a cause of increasing age.

The latencies of the ABR wave I peak appeared almost completely normal throughout the whole study, consistent with the hearing thresholds. Other studies have reported that prolonged latencies were only found in aging subjects if the hearing threshold was increased (Boettcher 2002), suggesting that the intensity levels of our noise exposures were not high enough to affect the ABR latencies. However, both noise and aging can cause changes in ABR amplitudes and eventually threshold shifts. It would be of interest to further explore possible structural differences at 


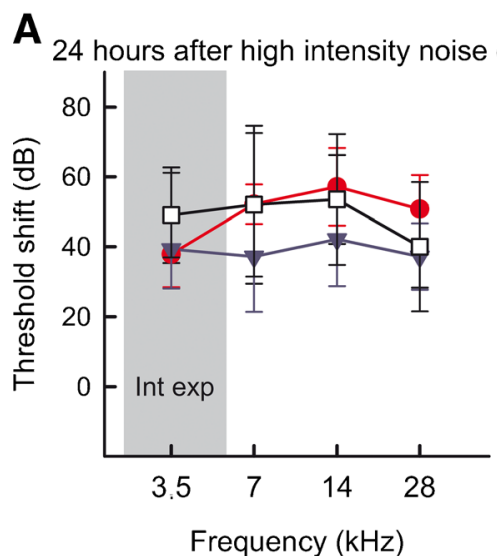

FIG. 6. $A B R$ threshold shifts after high-intensity noise exposure using a 4-h-long narrowband noise exposure of $110 \mathrm{~dB}$ SPL. A At $24 \mathrm{~h}$ after the acoustic overstimulation, the groups with previously non-exposed animals and animals repeatedly exposed at 101 and $104 \mathrm{~dB}$ SPL displayed threshold shifts of about $40-50 \mathrm{~dB}$. The group previously exposed at $104 \mathrm{~dB}$ SPL had slightly lower threshold shifts, but no significant differences were detected. B Two weeks after the

the level of the afferent nerve terminals after repeated moderate exposures.

Since the repeated noise exposures did not give rise to increased $A B R$ thresholds, we were interested in exploring possible changes in the animals' hearing using a more intense noise exposure. The thresholds were, as expected, increased $24 \mathrm{~h}$ after the exposure, and after 2 weeks, the threshold shifts were considered permanent for all groups (including the previously non-exposed control group). The highest threshold shift was found at frequencies close to the frequency span of the exposure-noise. The group previously exposed repeatedly to $104 \mathrm{~dB}$ SPL had slightly lower hearing thresholds than the other groups, which were significant at $28 \mathrm{kHz}$ compared to the non-exposed group, and moreover, at this frequency the thresholds had returned to normal. This group of animals had previously also displayed a decrease of ABR amplitudes later than the other groups. These results indicate that this intensity level of repeated moderate noise exposures made the animals somewhat more resistant to intense noise exposure later in life. Two types of "training" phenomena are described in the literature, both of them reducing inner ear susceptibility to noise. In one type, a low level of continuous non-damaging noise is used prior to a more intense noise exposure and is referred to in the literature as "sound conditioning" (Canlon et al. 1988; Yoshida and Liberman 2000). The second type, named "toughening," is observed following an interrupted time schedule, with exposure of animals to a noise that creates a temporary threshold shift (Miller and Watson 1959; Pukkila et al. 1997).

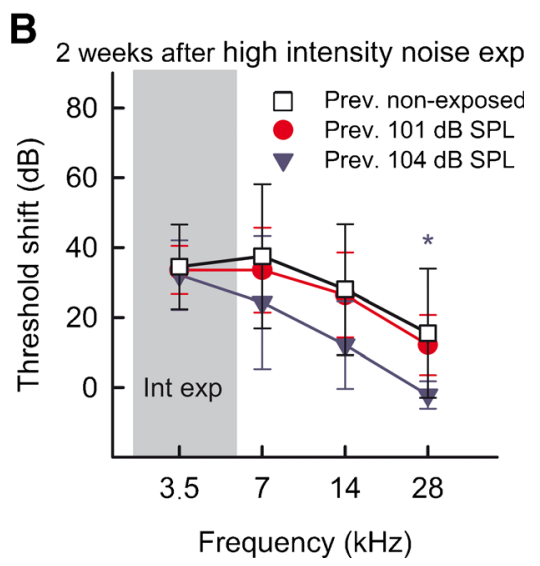

acoustic overstimulation, the threshold shifts had become permanent at about $20-40 \mathrm{~dB}$. The group previously exposed at $104 \mathrm{~dB}$ SPL displayed lower threshold shifts than the other groups, which were significant at $28 \mathrm{kHz}$ when compared to the previous non-exposed group. The area marked Int exp indicates the frequency span of the high-intensity noise exposure. ${ }^{*} P=0.013$ between the groups of previously non-exposed and exposed to $104 \mathrm{~dB}$ SPL.

Several exposures made the ears "toughened" resulting in smaller threshold shifts as the number of exposures increased. Neither sound conditioning nor toughening fully matches our experimental protocol with moderate 6-week interval of repeated noise exposures. Either intensity levels used in these studies were much lower (between 55 and $95 \mathrm{~dB}$ SPL) than in our exposures or the duration of the exposure were longer (typically for more than a week) with different repetition rates from days to several months. However, the mechanisms underlying sound conditioning or toughening may very well be the same as those involved in our model with repeated moderate noise exposures. One explanation for the beneficial effects could be triggering of several protective systems, for example the endogenous antioxidant system (Jacono et al. 1998) or production of neurotrophic factors (Nam et al. 2000), which are believed to protect cochlear structures from future damage.

The long-term auditory effects of non-damaging sound exposures throughout the life span are relatively unknown. The present animal model using repeated moderate noise exposures to mimic human early adulthood exposures may be used to explore these effects and their influence on age-related hearing loss. In daily life, individuals are regularly exposed to moderate sound levels during both working hours and leisure activities. The exposures may lead to a slight, often not detected, temporary hearing loss, which declines after a few days. Our observations indicate that these short-term effects do not impair hearing sensitivity. However, it should be noted that little is known of the long-term effects on 
onset and severity of age-related hearing loss. Other investigators have studied the effects of noise exposure at young age on age-related hearing loss (Kujawa and Liberman 2006, 2009), but they have primarily investigated the effect of a single and more intense noise exposure. In future studies, the present model could be used for studying age-related hearing loss in a more realistic situation. Possible intervention therapies may also be investigated in long-term studies using this model, aiming at better hearing preservation for the elderly.

\section{ACKNOWLEDGMENTS}

This work was supported by the Swedish Research Council (grant no. 2010-7209) and in part by funding from the European Union Seventh Framework Programme (FP7/ 2007-2013) under grant agreement no. 304925.

\section{Conflict of Interest}

The authors declare that they have no competing interests.

Open Access This article is distributed under the terms of the Creative Commons Attribution 4.0 International License (http://creativecommons.org/licenses/by/4.0/), which permits unrestricted use, distribution, and reproduction in any medium, provided you give appropriate credit to the original author(s) and the source, provide a link to the Creative Commons license, and indicate if changes were made.

\section{REFERENCES}

(NIDCD) NIoDaOCD (2010) http://www.nidcd.nih.gov/health/ statistics/Pages/quick.aspx

Altun M, Bergman E, Edstrom E, Johnson H, Ulfhake B (2007) Behavioral impairments of the aging rat. Physiol Behav 92(5):911-923. doi:10.1016/j.physbeh.2007.06.017

Alvarado JC, Fuentes-Santamaria V, Jareno-Flores T, Blanco Jl, Juiz JM (2012) Normal variations in the morphology of auditory brainstem response (ABR) waveforms: a study in Wistar rats. Neurosci Res 73(4):302-311. doi:10.1016/j.neures.2012.05.001

Boettcher FA (2002) Presbyacusis and the auditory brainstem response. J Speech Lang Hear Res JSLHR 45(6):1249-1261

Boettcher FA, Mills JH, Norton BL (1993) Age-related changes in auditory evoked potentials of gerbils. I. Response amplitudes. Hear Res 71(1-2):137-145

Browne CJ, Morley JW, Parsons CH (2012) Tracking the expression of excitatory and inhibitory neurotransmission-related proteins and neuroplasticity markers after noise induced hearing loss. PLoS One 7, e33272

Canlon B, Borg E, Flock A (1988) Protection against noise trauma by pre-exposure to a low level acoustic stimulus. Hear Res 34(2):197-200

CLARK WW (1991) Recent studies of temporary threshold shift (TTS) and permanent threshold shift (PTS) in animals. J Acoust Soc Am 90(1):155-163
Clark WW, Bohne BA, Boettcher FA (1987) Effect of periodic rest on hearing-loss and cochlear damage following exposure to noise. J Acoust Soc Am 82(4):1253-1264

Dalton DS, Cruickshanks KJ, Wiley TL, Klein BEK, Klein R, Tweed TS (2001) Association of leisure-time noise exposure and hearing loss. Audiology 40(1):1-9

Hamernik RP, Ahroon WA, Davis RI, Lei SF (1994) Hearing threshold shifts from repeated 6-h daily exposure to impact noise. J Acoust Soc Am 95(1):444-453

Hirose K, Liberman MC (2003) Lateral wall histopathology and endocochlear potential in the noise-damaged mouse cochlea. Jaro-J Assoc Res Otolaryngol 4:339-352

Jacono AA, Hu B, Kopke RD, Henderson D, Van De Water TR, Steinman HM (1998) Changes in cochlear antioxidant enzyme activity after sound conditioning and noise exposure in the chinchilla. Hear Res 117(1-2):31-38

Johnson AC, NyLen PR (1995) Effects of industrial solvents on hearing. Occup Med 10(3):623-640

Johnsson LG, Hawkins JE (1972) Sensory and neural degeneration with aging, as seen in microdissections of human inner-ear. Ann Otol Rhinol Laryngol 81(2):179-93

Konrad-Martin D, Dille MF, McMillan G, Griest S, McDermott D, FAusti SA, Austin DF (2012) Age-related changes in the auditory brainstem response. J Am Acad Audiol 23(1):18-35. doi:10.3766/jaaa.23.1.3

KuJAwa SG, LibERMAN MC (2006) Acceleration of age-related hearing loss by early noise exposure: evidence of a misspent youth. J Neurosci 26(7):2115-2123

KuJawa SG, Liberman MC (2009) Adding insult to injury: cochlear nerve degeneration after "temporary" noise-induced hearing loss. J Neurosci 29(45):14077-14085. doi:10.1523/ jneurosci.2845-09.2009

LenoIr M, Bock GR, Pujol R (1979) Supra-normal susceptibility to acoustic trauma of the rat pup cochlea. J De Physiologie $75(5): 521-524$

Liberman MC, Dodds LW (1984) Single-neuron labeling and chronic cochlear pathology. III. Stereocilia damage and alterations of threshold tuning curves. Hear Res 16:55-74

Lin HW, Furman AC, Kujawa SG, Liberman MC (2011) Primary neural degeneration in the Guinea pig cochlea after reversible noise-induced threshold shift. Jaro-J Assoc Res Otolaryngol 12(5):605-616. doi:10.1007/s10162-011-0277-0

Mannstrom P, Ulfhake B, Kirkegaard M, Ulfendahl M (2013) Dietary restriction reduces age-related degeneration of stria vascularis in the inner ear of the rat. Exp Gerontol 48(11):11731179. doi:10.1016/j.exger.2013.07.004

Miller JD, Watson CS (1959) Permanent deafness after continuous and spaced exposure to noise. J Acoust Soc Am 31(11):15741574

Mostafapour SP, Lahargoue K, Gates GA (1998) Noise-induced hearing loss in young adults: the role of personal listening devices and other sources of leisure noise. Laryngoscope 108(12):1832-1839. doi:10.1097/00005537-199812000-00013

Nam YJ, Stover T, Hartman SS, Altschuler RA (2000) Upregulation of glial cell line-derived neurotrophic factor (GDNF) in the rat cochlea following noise. Hear Res 146(1-2):1-6

Nordmann AS, Bohne BA, Harding GW (2000) Histopathological differences between temporary and permanent threshold shift. Hear Res 139(1-2):13-30

OHLEMILLER KK (2008) Recent findings and emerging questions in cochlear noise injury. Hear Res 245:5-17

Oіsнi N, Sснаснт J (2011) Emerging treatments for noise-induced hearing loss. Expert Opin Emerg Drugs 16(2):235-245. doi:10.1517/14728214.2011.552427

Pukkila M, Zhai S, Virkkala J, Pirvola U, Ylikoski J (1997) The "toughening" phenomenon in rat's auditory organ. Acta Otolaryngol Suppl 529:59-62 
Ruttiger L, Singer W, Panford-Walsh R, Matsumoto M, Lee SC, Zuccotti A, Zimmermann U, Jaumann M, Rohbock K, Xiong H, KNIPPER M (2013) The reduced cochlear output and the failure to adapt the central auditory response causes tinnitus in noise exposed rats. PLoS One 8, e57247

Rybak LP, Whitworth CA, Mukherjea D, Ramkumar V (2007) Mechanisms of cisplatin-induced ototoxicity and prevention. Hear Res 226(1-2):157-167. doi:10.1016/j.heares.2006.09.015

Saunders JC, Chen CS (1982) Sensitive periods of susceptibility to auditory trauma in mammals. Environ Health Perspect 44:63-66

SlePECKY N (1986) Overview of mechanical damage to the inner ear: noise as a tool to probe cochlear function. Hear Res 22:307-321
Stockwel CW, Ades HW, Engstrom H (1969) Patterns of hair cell damage after intense auditory stimulation. Ann Otol Rhinol Laryngol 78(6):1144-68

TORRe P, Fowler CG (2000) Age-related changes in auditory function of rhesus monkeys (Macaca mulatta). Hear Res 142(1-2):131-140. doi:10.1016/s0378-5955(00)00025-3

WANG Y, Ren CY (2012) Effects of repeated "benign" noise exposures in young CBA mice: shedding light on age-related hearing loss. Jaro-J Assoc Res Otolaryngol 13(4):505-515. doi:10.1007/s10162-012-0329-0

Wang Y, Hirose K, Liberman MC (2002) Dynamics of noise-induced cellular injury and repair in the mouse cochlea. Jaro 3:248-268

YoshidA N, Liberman MC (2000) Sound conditioning reduces noiseinduced permanent threshold shift in mice. Hear Res 148(12):213-219. doi:10.1016/s0378-5955(00)00161-1 